\title{
An approach to the management of fever of unknown origin in the setting of acute spinal cord injury
}

\author{
Brian R. Connolly ${ }^{1}$ Jeannie K. Harden ${ }^{1}$
}

Received: 1 November 2020 / Revised: 14 January 2021 / Accepted: 5 February 2021

(c) The Author(s), under exclusive licence to International Spinal Cord Society 2021

\begin{abstract}
Introduction Discovering the source of a patient's fever, in the absence of clinical localizing signs, can be a formidable undertaking. Without a clear symptom pattern to narrow the focus of a diagnostic workup, this task can require numerous noninvasive and invasive procedures, imaging studies, and laboratory tests to arrive at a conclusion. Identifying the source of a patient's fever in the setting of an acute spinal cord injury can present a unique challenge because this population is at risk for many different conditions that can cause fever, some of which are rarely seen in the general population.

Case presentation This case report describes a unique case and diagnostic workup of fever of unknown origin in a 51-year-old male with multiple medical comorbidities who presented to an inpatient rehabilitation service following an acute traumatic cervical spinal cord injury. Ultimately, it was determined that the patient had several different factors that were causing him to have intermittent fevers over a 5-week period.

Discussion This case demonstrates the importance of obtaining serial medical histories and physical examinations during the investigation for fever of unknown origin. It also supports why additional workup of a patient's fever may need to be considered even after a potential source is found. Finally, it demonstrates the need for increased awareness of quadriplegic fever so that this condition can be more effectively recognized, prevented, and managed in the future.
\end{abstract}

\section{Introduction}

Discovering the source of a patient's fever, in the absence of clinical localizing signs, can be a formidable undertaking. Without a clear symptom pattern to narrow the focus of a diagnostic workup, this task can require numerous noninvasive and invasive procedures, imaging studies, and laboratory tests to arrive at a conclusion. Since there are well over 200 different potential sources of fever [1], even the most extensive workups can end unsuccessfully. Indeed, it has been estimated that as many as $19-30 \%$ of fever workups conclude without a definitive fever etiology [2, 3]. In 1961, Vanderschueren et al. coined the term 'fever of unknown origin" (FUO) to describe such fevers [4]. In order to meet the original criteria for having a FUO, a patient was

Brian R. Connolly

connollyb@uthscsa.edu

1 Department of Physical Medicine and Rehabilitation, University of Texas Health Science Center at San Antonio, San Antonio, TX, USA required to have had intermittent fevers of $>101^{\circ} \mathrm{F}$ over a minimum period of 3 weeks without definitive cause after at least 1 week of in-hospital investigation [4]. In 1991, to account for advances in medical technology, the minimum duration of inpatient study was reduced to 3 days in the hospital or at least 3 hospital visits [5].

Identifying the source of a patient's fever following an acute spinal cord injury (SCI) can present a unique challenge. Not only is this population at increased risk for many of the most common causes of fever in the general populace including infections, deep venous thrombosis, pulmonary embolism, colitis, heterotopic ossification, and drug fevers [6], it is also prone to fever-causing conditions not commonly found in patients without damage to the central nervous system, which can add further uncertainty to a clinical picture. Such conditions include impairment to the autonomic system and physiologic adaptations that affect heat production and heat dissipation [7]. It is estimated that approximately half of patients experience a fever of known or unknown origin following an acute injury to the spinal cord [8]. Patients with a complete SCI, particularly in the cervical and thoracic regions, are more likely to experience a fever than are those with incomplete injuries [8]. 
In this manuscript we present a unique case and diagnostic workup of a patient with an acute SCI and multiple medical comorbidities that presented to our acute inpatient rehabilitation unit with intermittent fevers of unknown origin.

\section{Case presentation}

This case describes a 51-year-old male with a past medical history of multiple myeloma in full remission following autologous bone marrow transplant and chemotherapy, latent tuberculosis (TB), and diabetes mellitus type 2, who presented to an inpatient rehabilitation service after a fall from a chair that resulted in an injury to his spinal cord necessitating surgical decompression of the third through sixth cervical vertebrae (see Fig. 1 for the patient's magneticresonance imaging results). Prior to arrival, the patient had experienced intermittent fevers of up to $102.5^{\circ} \mathrm{F}$, each lasting no more than several hours, starting the day following his SCI and continuing after surgery over a period of 12 days. At the beginning of this period, he was treated with ciprofloxacin for complicated urinary tract infection after urine culture grew Enterococcus faecalis. However, it is of note that the urinalysis was found to have neither pyuria nor leukocyte esterase and was negative for nitrites. Shortly thereafter, antibiotic therapy was broadened when the patient continued to have intermittent fevers and computerized tomography (CT) imaging revealed hazy opacities in bilateral lung fields suggestive of infection. Despite the concern for pneumonia, the patient did not experience respiratory failure and never required mechanical ventilation support at any point during his hospital stay. CT imaging of the abdomen was not suggestive of intraabdominal infection.

Upon arrival to the acute rehabilitation unit on hospital day 20, antibiotic therapy had been completed and the patient had not had a fever over the previous $72 \mathrm{~h}$. The initial physical examination was significant for tetraplegia (C3 AIS C on the American Spinal Injury Association Impairment Scale). At the time of admission, complete blood count, metabolic panel and liver function parameters were within reference range.

On day 21, the patient's QuantiFERON-TB-Gold test was found to be positive. He also mounted a temperature of $100.1^{\circ} \mathrm{F}$ and was found to be diaphoretic with facial flushing. The infectious disease team was immediately consulted and determined that active TB was exceedingly unlikely given the lack of sputum production and absence of suggestive imaging findings. The positive lab result was concluded to be representative of latent TB. Over the next 4 days, the patient continued to experience high temperature readings up to $100.3^{\circ} \mathrm{F}$. He also had intermittent episodes of hypertension up to $190 / 83$ for which he was given multiple doses of hydralazine. After serial patient interviews, physical

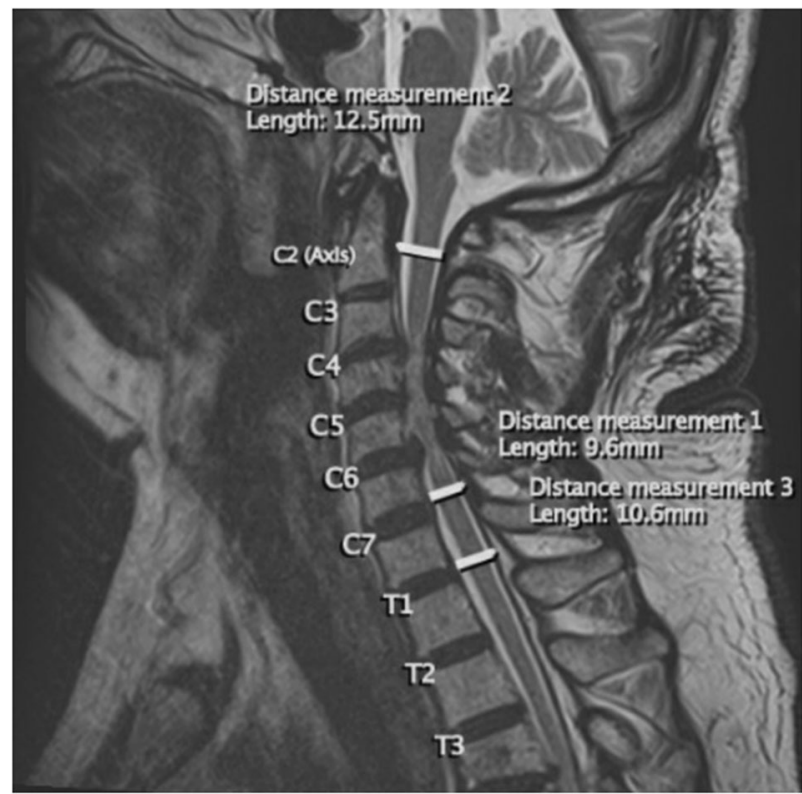

Fig. 1 Sagittal, T2 weighted MRI of the patient's cervical and upper thoracic vertebrae and surrounding structures. Initial MRI (magnetic resonance imaging) of the patient's cervical and upper thoracic vertebrae and surrounding structures upon arrival to the hospital. Thissagittal, T2 weighted image demonstrates severe cervical canal stenosis from C3 through C6 (as demonstrated by the spinal canal diameter measurements 1-3) with spinal cord compression and edema.

examinations, and watching for symptoms patterns, it was determined that the elevated blood pressure readings were due to chronic hypertension with intermittent episodes of autonomic dysreflexia as a result of bladder distension. The patient's indwelling foley catheter was replaced and antihypertensive medications adjusted accordingly, resulting in better blood pressure control.

On day 26, the patient developed a temperature of $100.9^{\circ} \mathrm{F}$, diaphoresis, increased muscle spasticity, and a non-productive cough and diarrhea. The infectious disease team was re-engaged, and once again ruled out active TB. Laboratory studies, including complete blood count, comprehensive metabolic panel, blood cultures, and urinalysis were negative or within normal limits apart from anemia and mild elevation of leukocyte esterase on urinalysis. There was low suspicion for multiple myeloma relapse because the patient did not have hypercalcemia, hyperproteinemia, elevated serum protein electrophoresis, urine protein electrophoresis, serum free light chain assay, or abnormal renal function, and CT imaging showed stable lytic lesions. Doppler ultrasound imaging of all extremities was negative for venous thrombosis. Heterotopic ossification was excluded from the differential as the patient was in the early stage of recovery from acute SCI and there was no clinical evidence to support this diagnosis. The fever resolved without treatment over the course of $1 \mathrm{~h}$. 
The next day, the patient had a fever of $101.7^{\circ} \mathrm{F}$. Given that the patient's previous urinalysis was borderline suggestive of infection, urinalysis and urine and blood cultures were repeated and the patient was started on empiric antibiotic therapy. The urinalysis would later show pyuria and bacteriuria and culture grew Klebsiella pneumoniae, for which the patient was treated with trimethoprimsulfamethoxazole based on antimicrobial susceptibility testing. Chest radiographs were without acute findings. Fungal serologies were found to be negative as were antinuclear antigen and rheumatoid factor tests. That afternoon, the patient's respiratory viral panel, which tested for 17 common viral respiratory illnesses and had been drawn the day before, returned positive for influenza B for which the patient was treated with oseltamivir phosphate. Of note, the patient was not tested for SARS-CoV-2 as this case occurred prior to the COVID-19 pandemic.

Over the next 4 days, the patient's vital signs gradually stabilized. The patient was discharged home in stable condition upon completion of his rehabilitation stay on hospital day 43 . He did not have any fevers over the week prior to discharge.

\section{Discussion}

Five days after admission to the acute rehabilitation unit, the patient met the criteria for FUO since the patient had experienced intermittent fevers for more than 3 weeks while admitted to a medical unit including his stay on the acute medicine service (see Table 1 for the patient's fever course). Although the patient was treated for a complicated urinary tract infection and pneumonia over this period, and there is evidence that injuries to the spinal cord can cause immunodepression [9], it was unclear whether these were active infections and the definitive source of the patient's fevers. Moreover, the patient continued to experience intermittent fevers despite treatment for these conditions.

Despite the patient's hospital length of stay and prior workup, a systematic approach was taken to perform a thorough investigation of the fever etiology, starting with a careful physical examination and medical history, both of which were vital to directing the fever workup. In formulating a differential diagnosis for a fever without an obvious source, an infectious etiology should be highly prioritized. In the setting of a SCI, infections of the respiratory system (e.g., pneumonia), genitourinary system (e.g., lower urinary tract infection), intraabdominal cavity (e.g., colitis or cholecystitis), skin (e.g., cellulitis) or bone (e.g., osteomyelitis) should be considered as neurologic injury may compromise normal motor and sensory function that serve as physiologic protective factors [6]. When a patient continues to have intermittent fevers despite evidence-based treatment for bacterial and viral infections, the treating physician should also be aware of the possibility of a fungal infection.

Common non-infectious etiologies of fever in a quadriplegic patient that should be considered include a deep venous thrombosis given the elevated risk secondary to acute immobilization and neurologic injury, heterotopic ossification, and drug fevers. Less common fever-inducing processes that merit consideration include malignancy (such as reactivation of multiple myeloma in this case), autoimmune disease, trauma, thyrotoxicosis, and metabolic disorders. For the patient in question, each of these was determined to be unlikely based on laboratory results and serial medical histories and physical examinations. Nuclear imaging, including positron emission tomography and scintigraphy, were not performed in this case although they could have been justified due to the patient's recurrent fevers, history of malignancy, and recent spine surgery. Finally, quadriplegic fever, a diagnosis of exclusion, may also be considered as a possible cause of fever in the setting of acute SCI.

The term quadriplegic fever was coined by Sugarman et al. in 1982 to describe periods of hyperthermia of unknown origin after acute traumatic SCI [6]. Such a fever occurs in $\sim 1$ of every $20-25$ patients who sustain an acute SCI, generally in the first few months after injury [8]. Despite the frequency of this condition, the mechanism by which it occurs has yet to be elucidated, although it is hypothesized that it is caused by disruption of various connecting pathways necessary for hypothalamic temperature regulation centers to function properly [8]. There is evidence that this condition should be considered following any fever following an acute SCI above the level of T6 [10]. In this case, dysautonomia likely contributed to the patient's intermittent fevers given that the patient had an acute cervical SCI and experienced orthostatic hypotension and autonomic dysreflexia throughout his hospital course.

Rather than having fevers of unknown etiology, the fevers of the featured patient were most likely caused by multiple known etiologies. Hospital acquired pneumonia, urinary tract infections, influenza B, and dysautonomia likely contributed to the intermittent elevation in temperatures observed over 5 weeks. In addition to the potentially catastrophic consequences that were prevented by treating acute fever-causing disease processes, a thorough investigation of all possible fever sources was an important part of the patient's rehabilitation, as even mild hyperthermia after SCI can compromise functional outcome and exacerbate tissue damage [11].

\section{Conclusion}

It is important to use a stepwise approach to the diagnosis of a fever, particularly when a patient's symptoms are fleeting or absent, when a patient has a rare medical 
Table 1 A chronological listing of the patient's temperature readings during his hospital stay.

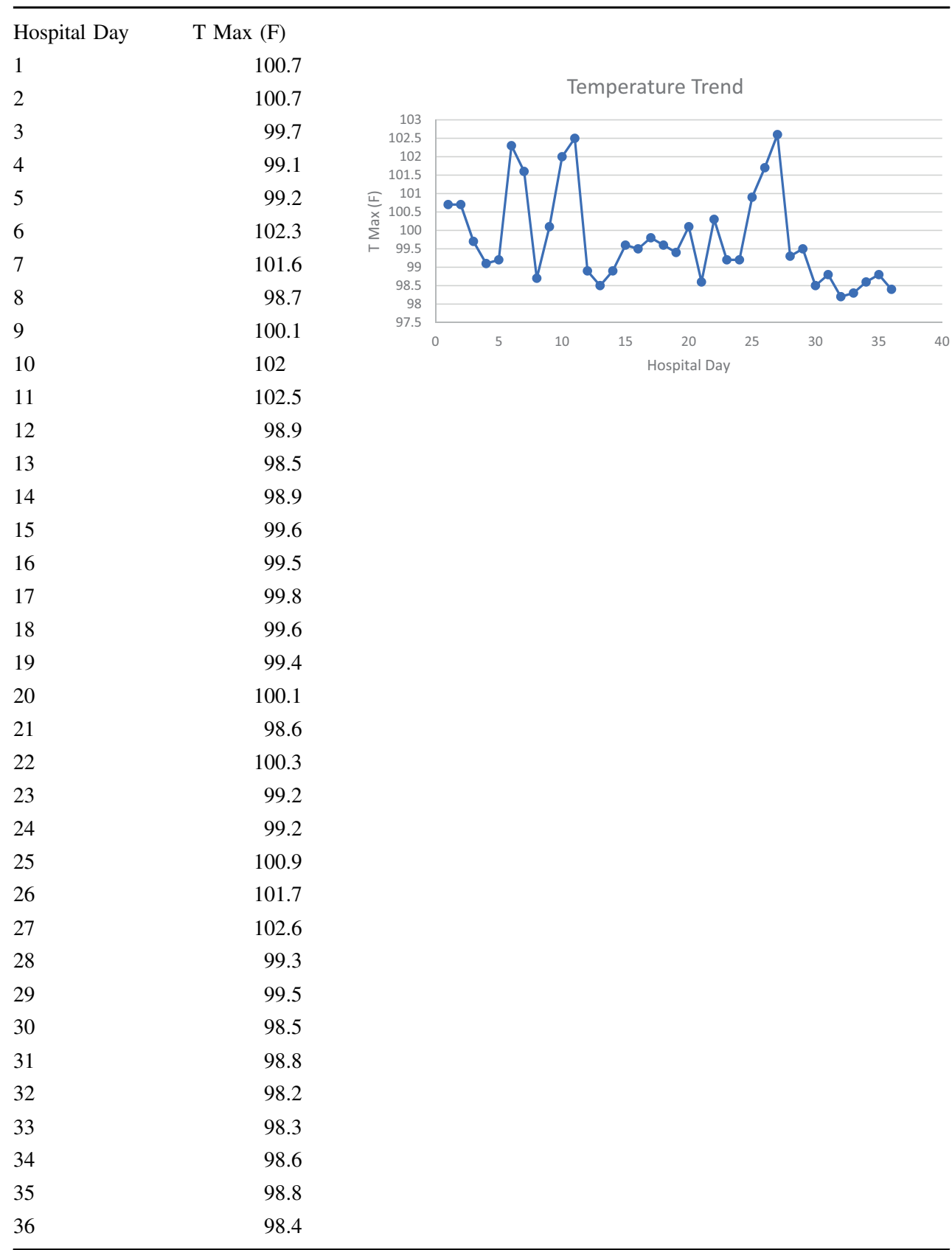

condition, and when a patient has multiple medical comorbidities. This case demonstrates the importance of obtaining serial medical histories and physical examinations during the investigation for FUO as these can produce critical diagnostic clues. Although clinical patterns may not be obvious at the outset, it is possible that a trend can be identified over a longer interval. When a potential source of fever is found, additional workup should still be considered, as multiple medical conditions can be the cause of a patient's fever. Finally, increased awareness of quadriplegic fever could help avoid unnecessary medical workup and antimicrobial treatment. The medical community should continue to study the incidence rate, clinical outcomes, and pathogenesis of this condition so that it can be more effectively recognized, prevented, and managed in the future.

\section{Compliance with ethical standards}

Conflict of interest The authors declare no competing interests.

Publisher's note Springer Nature remains neutral with regard to jurisdictional claims in published maps and institutional affiliations. 


\section{References}

1. Arnow PM, Flaherty JP. Fever of Unknown Origin. Lancet. 1997;350:575-80.

2. Vanderschueren S, Knockaert D, Adriaenssens T, Demey W, Durnez A, Blockmans D, et al. From prolonged febrile illness to fever of unknown origin: the challenge continues. Arch Intern Med. 2003;163:1033-41.

3. Mourad O, Palda V, Detsky A. A Comprehensive EvidenceBased Approach to Fever of Unknown Origin. Arch Intern Med. 2003;163:545.

4. Petersdorf RG, Beeson PB. Fever of unexplained origin: report on 100 cases. Medicine (Baltimore). 1961;40:1-30.

5. Durack DT, Street AC. Fever of unknown origin - reexamined and redefined. Curr Clin Top Infect Dis. 1991;11:35-51.
6. Sugarman B, Brown D, Musher D. Fever and infection in spinal cord injury patients. JAMA. 1982;248:66-70.

7. Price MJ, Trbovich M. Thermoregulation following spinal cord injury. Handb Clin Neurol. 2018;157:799-820.

8. Savage KE, Oleson CV, Schroeder GD, Sidhu GS, Vaccaro AR. Neurogenic fever after acute traumatic spinal cord injury: A qualitative systematic review. Global Spine J. 2016;6:607-14.

9. Held KS, Lane TE. Spinal cord injury, immunodepression, and antigenic challenge. Semin Immunol. 2014;26:415-20.

10. Colachis SC, Otis SM. Occurrence of fever associated with thermoregulatory dysfunction after acute traumatic spinal cord injury. Am J Phys Med Rehabil. 1995;74:114-9.

11. Yu CG, Jagid J, Ruenes G, Dietrich WD, Marcillo AE, Yezierski RP. Detrimental effects of systemic hyperthermia on locomotor function and histopathological outcome after traumatic spinal cord injury in the rat. Neurosurgery. 2001;49:152-8. 